\title{
ON EXISTENCE OF SOLUTION OF THE DIRICHLET PROBLEM OF FOURTH ORDER PARTIAL DIFFERENTIAL EQUATIONS WITH VARIABLE COEFFICIENTS*
}

\author{
BY \\ S. BALASUNDARAM AND P. K. BHATTACHARYYA \\ Indian Institute of Technology, New Delhi
}

\begin{abstract}
Sufficient conditions for the existence and uniqueness of the solution of the Dirichlet problem of fourth order elliptic partial differential equations with variable coefficients have been derived. In a number of examples of practical interest, the easy applicability of these results has been shown.

1. Introduction. A large class of problems of mathematical physics lead to fourth order elliptic partial differential equations with variable coefficients, the equations of the bending problems of elastic-isotropic, orthotropic and anisotropic-plates with variable (or constant) thickness being very particular cases of the general problem considered here. Hence, it is necessary to find sufficient conditions for the existence and uniqueness of the solution of the Dirichlet problem of these equations in weak form. This paper contains new results in this direction.
\end{abstract}

2. Notations. Let $\Omega$ be a domain in $\mathbb{R}^{2}$ with piecewise smooth boundary $\Gamma$ such that $\bar{\Omega}=\Omega \cup \Gamma$. Let $H^{m}(\Omega)$ be the usual Sobolev space $[1,4]$ of integral order $m \geqslant 0$ equipped with inner product $\langle\cdot, \cdot\rangle_{m, \Omega}$, norm $\|\cdot\|_{m, \Omega}$ and semi-norm $|\cdot|_{m, \Omega}$ such that $H^{0}(\Omega) \equiv$ $L^{2}(\Omega)$,

$$
H_{0}^{2}(\Omega)=\left\{v: v \in H^{2}(\Omega), \gamma_{0} v=\left.v\right|_{\Gamma}=0, \gamma_{1} v=\left.(\partial v / \partial n)\right|_{\Gamma}=0\right\} \equiv \overline{D(\Omega)},
$$

where $\gamma_{k}: H^{2}(\Omega) \rightarrow H^{2-k-1 / 2}(\Gamma)$ are trace operators with $k=0,1 ; H^{3 / 2}(\Gamma)$ and $H^{1 / 2}(\Gamma)$ being the fractional order Sobolev spaces on $\Gamma[1,4] ; D(\Omega)$ is the space of test functions on $\Omega[5]$.

3. The variational problem. To the Dirichlet problem (P) defined by: For given $f \in L^{2}(\Omega)$, find $u$ such that

$$
\Lambda u=f \quad \text { in } \Omega,\left.\quad u\right|_{\Gamma}=0,\left.\quad(\partial u / \partial n)\right|_{\Gamma}=0,
$$

\footnotetext{
* Received September 14, 1982.
} 
where

$$
\begin{aligned}
(\Lambda u)(x) & \equiv \frac{\partial^{2}}{\partial x_{k} \partial x_{l}}\left(a_{i j k l} \frac{\partial^{2} u}{\partial x_{i} \partial x_{j}}\right)(x) \\
& \equiv\left(a_{i j k l} u,{ }_{i j}\right)_{, k l}(x) \quad \text { for } x \in \Omega
\end{aligned}
$$

(in (3.2) and also in the sequel, the Einstein's summation convention has been followed), we associate the Galerkin Variational Problem $\left(\mathrm{P}_{\mathrm{G}}\right)$ defined by: Find $u \in H_{0}^{2}(\Omega)$ such that

$$
a(u, v)=l(v) \quad \forall v \in H_{0}^{2}(\Omega),
$$

where the continuous, symmetric bilinear form $a(\cdot, \cdot)$ and the continuous linear form $l(\cdot)$ are defined by: $\forall v, w \in H_{0}^{2}(\Omega)$,

$$
\begin{gathered}
a(v, w)=\langle\Lambda v, w\rangle_{0, \Omega}=\int_{\Omega} a_{i j k l} v,_{i j} w,_{k l} d \Omega=a(w, v), \\
l(v)=\langle f, v\rangle_{0, \Omega}=\int_{\Omega} f v d \Omega \quad \forall v \in H_{0}^{2}(\Omega) ;
\end{gathered}
$$

the coefficients $a_{i j k l}$ satisfy the following conditions: $\forall i, j, k, l=1,2$,

$$
a_{i j k l} \in C^{0}(\bar{\Omega}) ; \quad a_{i j k l}(x) \geqslant 0, \quad a_{i j k l}(x)=a_{k l i j}(x) \quad \forall x \in \bar{\Omega} .
$$

But without loss of generality, we can always assume that $\forall i, j, k, l=1,2$,

$$
a_{i j k l}(x)=a_{k l i j}(x)=a_{i j l k}(x)=a_{j i l k}(x) \quad \forall x \in \bar{\Omega}
$$

since if, for example, $a_{i j k l} \neq a_{j i k l}$ or $a_{i j k l} \neq a_{i j l k}$ (see Case (I) in Sec. 4.1) for some $i, j, k, l=1,2$, we can always define $\forall i, j, k, l=1,2$,

$$
\bar{a}_{i j k l}=\left(a_{i j k l}+a_{j i k l}+a_{j i l k}+a_{i j l k}\right) / 4
$$

such that $\forall i, j, k, l=1,2$,

$$
\bar{a}_{i j k l}(x)=\bar{a}_{k l i j}(x)=\bar{a}_{j i l k}(x)=\bar{a}_{j i k l}(x) \quad \forall x \in \bar{\Omega}
$$

and $\forall v, w \in H_{0}^{2}(\Omega)$,

$$
a_{i j k l} v,_{i j} w,_{k l}=\bar{a}_{i j k l} v,_{i j} w,_{k l}, \quad a(v, w)=a(w, v) .
$$

Now, we prove the main theorem on the $H_{0}^{2}(\Omega)$-ellipticity of a $(\cdot, \cdot)$.

THEOREM (3.1). If the coefficients $a_{i j k l}$ satisfy (A1)-(A2) and

$$
\begin{aligned}
& \inf _{x \in \bar{\Omega}}\left(a_{1111}-a_{1112}-a_{1122}\right)(x)>0, \\
& \inf _{x \in \bar{\Omega}}\left(a_{1212}-a_{1211}-a_{1222}\right)(x)>0, \\
& \inf _{x \in \bar{\Omega}}\left(a_{2222}-a_{2211}-a_{2212}\right)(x)>0,
\end{aligned}
$$

then $a(\cdot, \cdot)$ defined in $(3.4)$ is $H_{0}^{2}(\Omega)$-elliptic.

Proof. From (A2), we have $\forall x \in \bar{\Omega}, \forall \xi=\left(\xi_{11}, \xi_{12}, \xi_{21}, \xi_{22}\right) \in \mathbb{R}^{4}$ with $\xi_{21}=\xi_{12}$,

$$
\begin{aligned}
a_{i j k l}(x) \xi_{i j} \xi_{k l}= & \left(a_{1111}(x) \xi_{11}^{2}+2 a_{1122}(x) \xi_{11} \xi_{22}+a_{2222}(x) \xi_{22}^{2}\right) \\
& +4\left(a_{1211}(x) \xi_{12} \xi_{11}+a_{1212}(x) \xi_{12}^{2}+a_{2212}(x) \xi_{22} \xi_{12}\right) .
\end{aligned}
$$


$\forall x \in \bar{\Omega}, \forall \xi_{11}, \xi_{22} \in \mathbb{R}$

$$
\begin{aligned}
\left(a_{1111}(x) \xi_{11}^{2}+2 a_{1122}(x) \xi_{11} \xi_{22}+a_{2222}(x) \xi_{22}^{2}\right) \\
-\left(a_{1111}(x)+a_{2222}(x)-a_{1122}(x)\right)\left(\xi_{11}^{2}+\xi_{22}^{2}\right) \\
+\left(a_{1111}(x) \xi_{22}^{2}+a_{2222}(x) \xi_{11}^{2}\right) \\
=a_{1122}(x)\left(\xi_{11}+\xi_{22}\right)^{2} \geqslant 0 .
\end{aligned}
$$

Therefore, $\forall x \in \bar{\Omega}, \forall \xi_{11}, \xi_{22} \in \mathbb{R}$,

$$
\begin{aligned}
& a_{1111}(x) \xi_{11}^{2}+2 a_{1122}(x) \xi_{11} \xi_{22}+a_{2222}(x) \xi_{22}^{2} \\
& \geqslant\left(a_{1111}-a_{1122}\right)(x) \xi_{11}^{2}+\left(a_{2222}-a_{1122}\right)(x) \xi_{22}^{2}
\end{aligned}
$$

Similarly, the following inequalities can be established: $\forall x \in \bar{\Omega}, \forall \xi_{11}, \xi_{12}, \xi_{22} \in \mathbb{R}$,

$$
\begin{gathered}
\left(a_{1111}-a_{1122}\right)(x) \xi_{11}^{2}+2 a_{1112}(x) \xi_{11}\left(2 \xi_{12}\right)+a_{1212}(x)\left(2 \xi_{12}\right)^{2} \\
\geqslant\left(a_{1111}-a_{1112}-a_{1122}\right)(x) \xi_{11}^{2}+\left(a_{1212}-a_{1112}\right)(x)\left(2 \xi_{12}\right)^{2} \\
\left(a_{2222}-a_{2211}\right)(x) \xi_{22}^{2}+2 a_{1222}(x) \xi_{22}\left(2 \xi_{12}\right)+\left(a_{1212}-a_{1211}\right)(x)\left(2 \xi_{12}\right)^{2} \\
\geqslant\left(a_{2222}-a_{2211}-a_{2212}\right)(x) \xi_{22}^{2}+\left(a_{1212}-a_{1211}-a_{1222}\right)(x)\left(2 \xi_{12}\right)^{2} .
\end{gathered}
$$

Then, using (3.7)-(3.10), we have: $\forall x \in \bar{\Omega}, \forall \xi=\left(\xi_{11}, \xi_{12}, \xi_{21}, \xi_{22}\right) \in \mathbb{R}^{4}$ with $\xi_{21}=\xi_{12}$,

$$
\begin{aligned}
& a_{i j k l}(x) \xi_{i j} \xi_{k l} \geqslant\left(a_{1111}-a_{1112}-a_{1122}\right)(x) \xi_{11}^{2} \\
& \quad+\left(a_{1212}-a_{1211}-a_{1222}\right)(x)\left(2 \xi_{12}\right)^{2}+\left(a_{2222}-a_{2211}-a_{2212}\right)(x) \xi_{22}^{2} \\
& \Rightarrow a(v, v)=\int_{\Omega} a_{i j k l}(x) v,_{i j} v,_{k l} d \Omega \\
& \geqslant \int_{\Omega}\left[\left(a_{1111}-a_{1112}-a_{1122}\right)(x)\left(v,{ }_{11}\right)^{2}\right. \\
& \quad+4\left(a_{1212}-a_{1211}-a_{1222}\right)(x)\left(v,{ }_{12}\right)^{2} \\
& \left.\quad+\left(a_{2222}-a_{2212}-a_{2211}\right)(x)\left(v,,_{22}\right)^{2}\right] d \Omega \\
& \geqslant \alpha_{0} \int_{\Omega} v,_{i j} v,_{i j} d \Omega=\alpha_{0}|v|_{2, \Omega}^{2} \quad \forall v \in H_{0}^{2}(\Omega),
\end{aligned}
$$

where

$$
\begin{aligned}
& \alpha_{0}=\min \left\{\inf _{x \in \bar{\Omega}}\left(a_{1111}-a_{1112}-a_{1122}\right)(x),\right. \\
& \left.\quad 2 \inf _{x \in \bar{\Omega}}\left(a_{1212}-a_{1211}-a_{1222}\right)(x), \inf _{x \in \bar{\Omega}}\left(a_{2222}-a_{2211}-a_{2212}\right)(x)\right\}>0 .
\end{aligned}
$$

Then, the result follows from the application of the Poincare-Friedrichs inequality [4]. 
Now, if

$$
\begin{aligned}
& \inf _{x \in \bar{\Omega}}\left(a_{1111}-a_{1112}-a_{1122}\right)(x)=0, \\
& \inf _{x \in \bar{\Omega}}\left(a_{1212}-a_{1211}-a_{1222}\right)(x)=0, \\
& \inf _{x \in \bar{\Omega}}\left(a_{2222}-a_{2211}-a_{2212}\right)(x)=0 .
\end{aligned}
$$

(See Case (II) in the Sec. (4.1)), then the Theorem (3.1) is not applicable. But we have TheOREM (3.2). If $\forall i, j, k, l=1,2$,

$$
a_{i j k l}=A_{i j k l}+\beta_{i j k l}
$$

where $\forall i, j, k, l=1,2, A_{i j k l}$ satisfy the conditions (A1)-(A2) and (3.6) and $\beta_{i j k l}$ satisfy

$$
\int_{\Omega} \beta_{i j k l} v,{ }_{i j} v,{ }_{k l} d \Omega \geqslant 0 \quad \forall v \in H_{0}^{2}(\Omega),
$$

then $a(\cdot, \cdot)$ is $H_{0}^{2}(\Omega)$-elliptic.

Proof. Using (3.11), we obtain: $\forall v \in H_{0}^{2}(\Omega)$,

$$
\begin{aligned}
a(v, v) & =\int_{\Omega}\left(A_{i j k l}+\beta_{i j k l}\right) v,_{i j} v,_{k l} d \Omega \\
& \geqslant \int_{\Omega} A_{i j k l} v,_{i j} v,_{k l} d \Omega
\end{aligned}
$$

(by virtue of (3.12)), from which the result follows by the Theorem (3.1), since $A_{i j k l}$ satisfy (A1)-(A2) and (3.6).

Remark (3.1). $\beta_{i j k l}$ in (3.11) do not satisfy (A1)-(A2) and (3.6) in general.

THEOREM (3.3). If the coefficients $a_{i j k l}$ satisfy (A1)-(A2) and (3.6) (resp. (3.11) and (3.12)), the problem $\left(\mathrm{P}_{\mathrm{G}}\right)$ has a unique solution.

Proof. The result follows from the Theorem (3.1) (resp. Theorem (3.2)) and the Lax-Milgram lemma [4].

Remark (3.2). Since $a(\cdot, \cdot)$ is symmetric, the Ritz variational problem $\left(\mathrm{P}_{\mathrm{R}}\right)$ corresponding to $(P)$ can be defined as follows: For given $f \in L^{2}(\Omega)$, find $u \in H_{0}^{2}(\Omega)$ such that

$$
J(u)=\inf _{v \in H_{0}^{2}(\Omega)} J(v),
$$

where

$$
J(v)=\frac{1}{2} a(v, v)-l(v) \quad \forall v \in H_{0}^{2}(\Omega) .
$$

Proposition (3.1). If $a_{i j k l}$ satisfy (A1)-(A2) and (3.6) (resp. (3.11)-(3.12)) and $u \in H_{0}^{2}(\Omega)$ is the solution of $\left(\mathrm{P}_{\mathrm{G}}\right)$, then $u \in H_{0}^{2}(\Omega)$ is also the unique solution of $\left(\mathrm{P}_{\mathrm{R}}\right)$.

4. Examples. First of all, we shall consider the biharmonic problem whose results are well known and then the bending problems of elastic-isotropic, orthotropic and anisotropic-plates with constant and variable thickness in order to illustrate the generality of the results obtained. In all the examples given below, only the $H_{0}^{2}(\Omega)$-ellipticity of the 
corresponding bilinear form $a(\cdot, \cdot)$ has been proved by Theorem (3.1) (resp. Theorem (3.2)), since the existence and uniqueness of the solution $u \in H_{0}^{2}(\Omega)$ of the corresponding problem $\left(\mathrm{P}_{\mathrm{G}}\right)$ follows immediately from the Theorem (3.3).

\subsection{The biharmonic problem.}

Case (I). For $a_{i j k l}=\delta_{i k} \delta_{j l}, \Lambda u=\Delta \Delta u$. But $a_{i j k l} \neq a_{j i k l}, a_{i j k l} \neq a_{i j l k}$ in general, i.e. (A2) is not satisfied, although (Al) holds, since $a_{i j k l}=a_{k l i j}$. Define $\bar{a}_{i j k l}$ by (3.5) such that $\bar{a}_{i i i i}=1, \bar{a}_{1212}=\bar{a}_{1221}=\bar{a}_{2112}=\bar{a}_{2121}=\frac{1}{2}, \bar{a}_{i j k l}=0$ otherwise. Then, $\bar{a}_{i j k l}$ satisfy (A1)(A2) and also (3.6), since $\bar{a}_{1111}-\bar{a}_{1112}-\overline{\mathrm{a}}_{1122}=1, \bar{a}_{1212}-\bar{a}_{1211}-\bar{a}_{1222}=\frac{1}{2}, \bar{a}_{2222}-$ $\bar{a}_{2211}-\bar{a}_{2212}=1$, and the $H_{0}^{2}(\Omega)$-ellipticity of $a(\cdot, \cdot)$ defined by

$$
a(v, w)=\int_{\Omega} \delta_{i k} \delta_{j l} v,{ }_{i j} w,{ }_{k l} d \Omega=\int_{\Omega} v,{ }_{i j} w,{ }_{i j} d \Omega
$$

follows from the Theorem (3.1).

Case (II). $a_{i j k l}=\delta_{i j} \delta_{k l}$ yield $\Lambda u=\Delta \Delta u$ and $a(v, w)=\int_{\Omega} \Delta u \Delta w d \Omega$. Then $a_{i j k l}$ satisfy (A1)-(A2), but not (3.6), since $a_{1111}-a_{1112}-a_{1122}=a_{1212}-a_{1211}-a_{1222}=a_{2222}$ $a_{2211}-a_{2212}=0$. Hence, Theorem (3.1) is not applicable. In order to apply the Theorem (3.2), define $\delta_{i j} \delta_{k l}=\bar{a}_{i j k l}+\beta_{i j k l}$, where $\bar{a}_{i j k l}$ are those given above in Case (I) which satisfy (A1)-(A2) and (3.6), and $\beta_{i j k l}$ are given by: $\beta_{1122}=\beta_{2211}=1, \beta_{1212}=\beta_{2112}=$ $\beta_{2121}=\beta_{1221}=-\frac{1}{2}, \beta_{i j k l}=0$ otherwise (see Remark (3.1)). $\forall v \in D(\Omega)$,

$$
\int_{\Omega} \beta_{i j k l} v,{ }_{i j} v,{ }_{k l} d \Omega=2 \int_{\Omega}\left(v,{ }_{11} v,{ }_{22}-\left(v,{ }_{12}\right)^{2}\right) d \Omega=0 .
$$

Since $D(\Omega)$ is dense in $H_{0}^{2}(\Omega), \beta_{i j k l}$ satisfy (3.12). Now, the $H_{0}^{2}(\Omega)$-ellipticity of $a(\cdot, \cdot)$ follows from Theorem (3.2).

4.2 Bending problems of elastic plates. The bending problem of a clamped thin elastic plate is defined by (P) of the corresponding plate operator $\Lambda$ given by (3.1), where $u=u\left(x_{1}, x_{2}\right)$ denotes the normal deflection at any point $\left(x_{1}, x_{2}\right)$ of the middle plane $\bar{\Omega}$ of the elastic plate, $\Gamma$ being its boundary along which the plate is clamped, the coefficients $a_{i j k l}$ denote elastic properties and thickness of the plate, $f \in L^{2}(\Omega)$ denotes the load function. We shall consider elastic plates first with constant thickness and then with variable thickness.

a) Plates with thickness $h=$ constant.

(I) For anisotropic case [3], $a_{i i i i}=D_{i i}, a_{1212}=a_{1221}=a_{2121}=a_{2112}=D_{66}, a_{1112}=a_{1121}$ $=a_{1211}=a_{2111}=D_{16}, a_{1222}=a_{2122}=a_{2212}=a_{2221}=D_{26}$ and $a_{2211}=a_{1122}=D_{12}$, where $D_{i j}$ denote rigidities [3] having the properties: $D_{11}, D_{22}, D_{66}>0 ; D_{12}=\nu_{1} D_{22}=\nu_{2} D_{11}$, $0 \leqslant \nu_{i}<\frac{1}{2}(i=1,2) ; 0 \leqslant D_{16}<\left(1-\nu_{2}\right) D_{11} ; 0 \leqslant D_{26}<\left(1-\nu_{1}\right) D_{22}$ and $D_{16}+D_{26}<$ $D_{66}$; and the Anisotropic plate operator $\Lambda$ is given by: $\Lambda u=D_{11} u,_{1111}+4 D_{16} u,_{1112}+$ $2\left(D_{12}+2 D_{66}\right) u,_{1122}+4 D_{26} u,_{1222}+D_{22} u,{ }_{2222}$. The coefficients $a_{i j k l}$ satisfy (A1)-(A2) and also (3.6), since $a_{1111}-a_{1112}-a_{1122}=D_{11}\left(1-\nu_{2}\right)-D_{16}>0, a_{1212}-a_{1211}-a_{1222}$ $=D_{66}-\left(D_{16}+D_{26}\right)>0, a_{2222}-a_{2212}-a_{2211}=D_{22}\left(1-\nu_{1}\right)-D_{26}>0$. Hence, the $H_{0}^{2}(\Omega)$-ellipticity of the bilinear form $a(\cdot, \cdot)$ defined by 


$$
\begin{aligned}
a(v, w)=\int_{\Omega}\left[\left(D_{11} v,_{11}+\right.\right. & \left.2 D_{16} v,_{12}+D_{12} v,_{22}\right) w,_{11} \\
+ & 2\left(D_{16} v,_{11}+\right. \\
+ & \left.2 D_{66} v,_{12}+D_{26} v,_{22}\right) w,_{12} \\
& \left.+\left(D_{12} v,_{11}+2 D_{26} v,_{12}+D_{22} v,_{22}\right) w,_{22}\right] d \Omega
\end{aligned}
$$

follows from the Theorem (3.1).

(II) For orthotropic case $[2,3,6,7], a_{i i i i}=D_{i}$,

$$
\begin{aligned}
& a_{1122}=a_{2211}=D_{12}=\nu_{1} D_{2}=\nu_{2} D_{1}, \\
& a_{1212}=a_{1221}=a_{2112}=a_{2121}=\left(H-\nu_{2} D_{1}\right) / 2, \\
& a_{1211}=a_{1112}=a_{1121}=a_{2111}=0, \\
& a_{2212}=a_{1222}=a_{2122}=a_{2221}=0,
\end{aligned}
$$

where

$$
\begin{aligned}
D_{i} & =E_{i} h^{3} /\left(12\left(1-\nu_{1} \nu_{2}\right)\right), \\
H & =D_{12}+2 D_{t}, D_{t}=G h^{3} / 12, \\
G & =E_{1} E_{2} /\left(E_{1}+\left(1+2 \nu_{1}\right) E_{2}\right)=E_{1} E_{2} /\left(E_{2}+\left(1+2 \nu_{2}\right) E_{1}\right), \\
E_{1} \nu_{2} & =E_{2} \nu_{1},
\end{aligned}
$$

$E_{i}$ and $\nu_{i}(i=1,2)$ are Young's moduli and Poisson's coefficients respectively. Then, the corresponding Orthotropic plate operator $\Lambda$ is defined by $\Lambda u=D_{1} u,_{1111}+2 H u,_{1122}+$ $D_{2} u,{ }_{2222}$. The coefficients $a_{i j k l}$ satisfy (A1)-(A2) and also (3.6), since

$$
\begin{aligned}
& a_{1111}-a_{1112}-a_{1122}=D_{1}\left(1-\nu_{2}\right)>0 \\
& a_{1212}-a_{1211}-a_{1222}=D_{t}>0 ; \\
& a_{2222}-a_{2211}-a_{2212}=\left(1-\nu_{1}\right) D_{2}>0 .
\end{aligned}
$$

Hence, the $H_{0}^{2}(\Omega)$-ellipticity of the corresponding bilinear form $a(\cdot, \cdot)$ defined by

$$
\begin{aligned}
a(v, w)=\int_{\Omega}\left[\left(D_{1} v,{ }_{11}+\nu_{2} D_{1} v,_{22}\right) w,{ }_{11}\right. & +2\left(H-\nu_{2} D_{1}\right) v,_{12} w,_{12} \\
& \left.+\left(\nu_{2} D_{1} v,_{11}+D_{2} v,_{22}\right) w,,_{22}\right] d \Omega
\end{aligned}
$$

follows from the Theorem (3.1).

(III) For isotropic case [3,6,7], which is obtained from the orthotropic case in (II) by putting $E_{1}=E_{2}=E$ and $\nu_{1}=\nu_{2}=\nu$ in (4.1) and (4.2) such that $D_{1}=D_{2}=H=D$, the Isotropic plate operator $\Lambda$ is defined by: $\Lambda u \equiv D \Delta \Delta u$, and the $H_{0}^{2}(\Omega)$-ellipticity of the corresponding bilinear form $a(\cdot, \cdot)$ defined by

$$
a(v, w)=\int_{\Omega} D\left[\left(v,_{11}+\nu v,_{22}\right) w,_{11}+2(1-\nu) v,_{12} w,_{12}+\left(\nu v,_{11}+v,_{22}\right) w,_{22}\right] d \Omega
$$

follows from (4.1)-(4.3) and Theorem (3.1).

b) Plates with variable thickness $h=h\left(x_{1}, x_{2}\right)>0$ : The thickness function $h$ satisfies the following condition: 


$$
h \varepsilon C^{0}(\bar{\Omega}), \quad h_{0}=\min _{\left(x_{1}, x_{2}\right) \in \bar{\Omega}} h\left(x_{1}, x_{2}\right)>0 .
$$

For the sake of brevity, we shall consider only the isotropic plates with variable thickness, since the proofs for the orthotropic and anisotropic plates with variable thickness $h$ satisfying (A3) are similar. For the isotropic case, $a_{i i i i}=D, a_{1122}=a_{2211}=\nu D, a_{1212}=$ $a_{1221}=a_{2121}=a_{2112}=D(1-\nu) / 2$, and $a_{i j k l}=0$ otherwise, where

$$
D=D\left(x_{1}, x_{2}\right)=\left(E h^{3} /\left(12\left(1-\nu^{2}\right)\right)\right)\left(x_{1}, x_{2}\right) \geqslant E h_{0}^{3}\left(12\left(1-\nu^{2}\right)\right)>0 \text {. }
$$

Then $a_{i j k l}$ satisfy (A1)-(A2) and (3.6), since $a_{1111}-a_{1112}-a_{1122}=(1-\nu) D>0, a_{1212}$ $-a_{1211}-a_{1222}=D(1-\nu) / 2>0, a_{2222}-a_{2211}-a_{2212}=(1-\nu) D>0$. Hence, the corresponding bilinear form $a(\cdot, \cdot)$ defined by $(4.5)$, in which $D$ is given by (4.6), is $H_{0}^{2}(\Omega)$-elliptic by Theorem (3.1).

\section{BIBLIOGRAPHY}

[1] R. A. Adams, Sobolev spaces, Academic Press, New York, 1975.

[2] M. T. Huber, Probleme der Statik technische wichtiger orthotroper Platten, Warsaw, 1929

[3] S. G. Lekhnitskii, Anisotropic plates, Gordon and Breach, New York, 1968

[4] J. L. Lions, Problèmes aux limites dans les équations dérivées partielles, Les presses de L'Université de Montréal, Montreal, 1965

[5] L. Schwartz, Theorie des distributions, Hermann, Paris, 1966

[6] S. Timoshenko and S. Woinowsky-Krieger, Theory of plates and shells, McGraw-Hill, New York, 1955

[7] M. S. Troitsky, Stiffened plates, Elsevier, New York, 1976 